\title{
Gestión y rehabilitación energética de edificios existentes: procedimiento experimental de diagnosis y caracterización energética
}

\author{
Management and rebuilding energy of existing buildings: diagnostic and characterization \\ procedure energy from measured data
}

\author{
Julián Arco (Autor Principal) \\ juliannn@ugr.es \\ Campus de Fuentenueva \\ 18071- Granada - España \\ José Sánchez (Autor Oficial Contacto) \\ Universidad de Sevilla, España \\ jsr@us.es
}

Grupo de Investigación Optimización Computacional en Comunicaciones e Ingeniería, Escuela Técnica Superior de Ing. de Edificación, Universidad de Granada, España (+34) 958241000 (20022) fax (+34) 958248990

\author{
Raúl Saucedo \\ Universidad de Granada, España \\ saucedo@ugr.es
}

\author{
Francisco Gil Montoya \\ Universidad de Almería, España \\ pagilm@ual.es \\ José Manuel Salmerón \\ Universidad de Sevilla, España \\ jms@us.es \\ José Luis Molina \\ Universidad de Sevilla, España \\ jlmolina@us.es
}

\begin{abstract}
Resumen
Uno de los factores esenciales para la gestión y rehabilitación energética de edificios existentes es establecer un procedimiento práctico y eficiente, a partir del control distribuido y de la monitorización inteligente, tratando de automatizar los procesos de funcionamiento, conocer el estado de las instalaciones, monitorizar las variables críticas y recibir información on-line y off-line del rendimiento de las mismas con el fin de asegurar las condiciones de confort en un contexto de consumo de energía racional. Todo ello se concretiza en el desarrollo de un nuevo modelo para la predicción del consumo energético de edificios. Esta predicción se realiza en dos niveles: horario, con un modelo derivado de las funciones de transferencia aplicadas a la edificación, y diario, con un modelo simplificado a partir del anterior. El objetivo es conseguir un consumo inteligente de energía, y sobre todo eficiente a través de la implementación progresiva de medidas de ahorro de energía
\end{abstract}

\author{
Código: 0131 \\ Fecha de Aceptación: 1 de diciembre de 2013
}

Keywords: Rehabilitation energy; Savings verification; Monitoring of buildings; energy services.

\section{Introducción}

Desde la Universidad de Granada y bajo el prisma de una especial sensibilidad por la eficiencia energética de sus edificios, se plantean los siguientes objetivos con el control distribuido: Tener un autoconocimiento del uso de la energía y de cuál es el potencial de ahorro y de mejora. Gestionar la energía de forma activa con documentación ordenada y registros fiables de ahorros conseguidos. Tanto a nivel general (i.e. climatización de los distintos edificios, iluminación...) como particular (i.e. estudio del consumo energético en un laboratorio en particular). Identificar y priorizar los aspectos energéticos.

Establecer objetivos de mejora de la eficiencia y optimización de la energía. Establecer procedimientos de control y seguimiento de los consumos de energía. Establecer protocolos de auditorías energéticas en la Universidad. Tener un registro de resultados de las mejoras implementadas accesible a cualquiera que lo desee. Permitir el acceso a los resultados de las mejoras implementadas para y como recurso para todos los departamentos que quieran hacer uso de ellos. Publicitar la buena gestión de la energía que hace la Universidad a través de la web de la oficina. Tener comunicación con los usuarios de las instalaciones para calificar la bondad de la medida implementada.

Las arquitecturas de control tradicionales, aplicadas a un solo edificio que se explota de manera aislada, proporcionan una solución adecuada y satisfactoria siempre que se conciba el edificio como un sistema cerrado y autocontenido. Por otra parte, cuando el objetivo es el de gestionar un conjunto de edificios (Campus Universitario), el problema que se plantea presenta diferencias cruciales en diversos aspectos y por tanto, 
será diferente también su solución. Comparadas con las arquitecturas de control tradicionales, las arquitecturas multiedificio integradas son soluciones que en la práctica han demostrado proporcionar un rendimiento considerablemente mayor y un costo de implementación y explotación sensiblemente menor que su equivalente monoedificio.

Las ideas principales sobre la que se sustentan las arquitecturas multiedificio Integradas son principalmente dos: Independización de los Niveles de Gestión, Control y Presentación: Consiste en separar los tres niveles básicos que conforman un sistema de control. Redistribución de Bloques Funcionales: Consiste en identificar y distribuir adecuadamente en diferentes elementos software $y$ hardware los bloques funcionales presentes en los sistemas de control tradicionales. Con la aplicación de las dos soluciones anteriores, se consigue maximizar los parámetros básicos de un Sistema de Control de Edificios: Robustez, Potencia, Facilidad de Explotación y Flexibilidad.

\section{Caracterización inversa del comportamiento energético real: verificación de ahorros}

\section{Descripción}

Fundamentalmente se establece el desarrollo de un nuevo modelo para la predicción del consumo energético de edificios. Esta predicción se realiza en dos niveles: horario, con un modelo derivado de las funciones de transferencia aplicadas a la edificación, y diario, con un modelo simplificado a partir del anterior.

Dentro de un proyecto de rehabilitación energética, este modelo se hace necesario para la verificación de ahorros. Al implantarse una medida de mejora en un edificio, cambia su comportamiento y se pierde la situación de referencia (línea base). Para poder calcular el ahorro conseguido con dicha mejora, debe compararse el consumo de energía actual y el que se habría tenido si la medida no se hubiera llevado a cabo. Así, un modelo predictivo permite proyectar el uso de energía en el edificio desde un periodo de referencia, que puede ser medido, a un periodo posterior. Esta proyección requiere el ajuste de la línea base a diferentes condiciones de clima, ocupación, u otras variables que puedan afectar al consumo de energía.

En general, dentro del esquema habitual para la identificación de modelos, el primer paso es determinar la forma apropiada del modelo, con un sentido físico consistente. Si en algún momento se requieren hipótesis o simplificaciones, éstas deben estar suficientemente justificadas. El segundo paso es elegir una metodología estadística apropiada para el ajuste de los parámetros desconocidos, es decir, para la identificación de los coeficientes a partir de los datos disponibles.

Así, el tercer paso es el diseño de un plan de experimentos de calidad. La medición es un punto clave puesto que los valores que se obtengan serán los inputs del modelo. La calidad de los sensores, su revisión e inspección continua son de vital importancia.

Este proceso puede esquematizarse en la siguiente figura. En ella se incluye además una fase de validación, en la que mediante nuevos datos experimentales se corrobora la validez y el rango de aplicación del modelo. Si el modelo no satisface unas ciertas premisas en cuanto a su precisión, debe revisarse el proceso de forma iterativa.

El siguiente epígrafe describe el procedimiento que permite determinar el modelo predictivo en base de tiempo diaria. Aunque cuanto menor sea la base de tiempo mayor es la precisión del modelo, la utilidad del uso de una base de tiempo diaria puede justificarse con los siguientes argumentos: Cuando se busca una opción más simplificada pero igual de robusta que el modelo horario., por ejemplo cuando el objetivo perseguido es la creación de la situación de referencia energética del edificio o sistema antes de su mejora (baseline en EVO). Cuando no es posible llegar a un nivel suficientemente detallado de todos los parámetros, por ejemplo cuando se pretende caracterizar un conjunto de sistemas o subsistemas pertenecientes al edificio, o el edificio completo. Cuando el objetivo es la calibración de programas de simulación energética. Si se quiere ir al detalle en un elemento en particular sí tiene sentido el modelo horario, pero si se busca una visión más global el modelo diario es más adecuado. Cuando el objetivo es el cálculo de integrales mensuales o estacionales de las demandas o consumos energéticos del edificio.

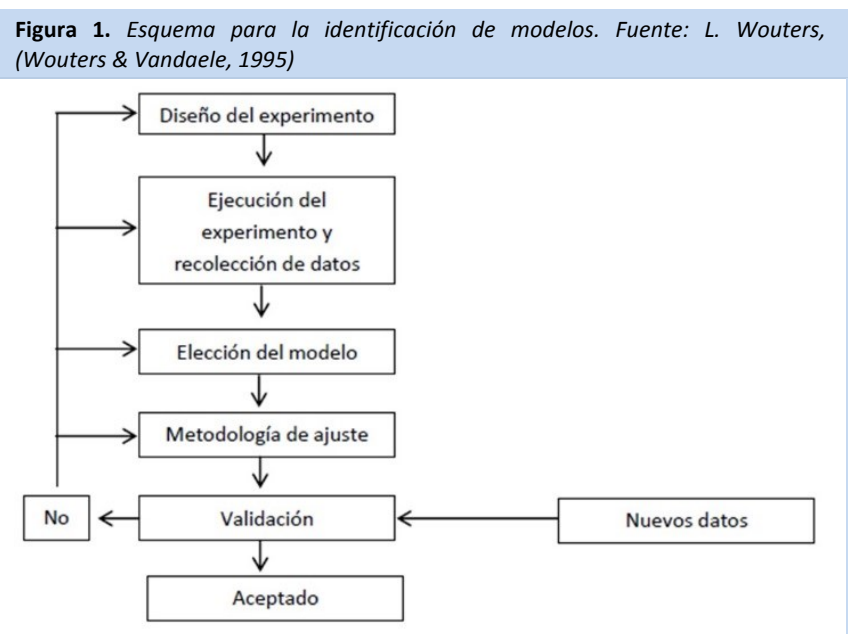

Partiendo del modelo en base horaria detallado anteriormente, puede extrapolarse directamente el modelo en base diaria, sin más que modificar la base de tiempo, o bien puede deducirse un modelo diario con el mismo fundamento pero simplificado al asumir varias hipótesis. Una vez que se ha demostrado la necesidad de modelos capaces de predecir el consumo de energía en edificios bajo unas condiciones dadas, cabe preguntarse qué tipo de métodos se emplean hoy por hoy con este fin.

En la referencia (Hai-xiang \& Magoulès, 2012), se realiza un trabajo de investigación de los diferentes modelos predictivos del consumo de energía en edificios actuales. Dichos modelos abarcan desde el proceso de diseño del edificio a su operación o modificación, así como desde el análisis de subsistemas hasta el modelado a nivel regional o nacional. Además, las predicciones pueden realizarse analizando a fondo cada factor de influencia o simplemente considerando algunos factores importantes. 
El comportamiento energético de un edificio está influenciado por diversos factores, como las condiciones climáticas (especialmente la temperatura seca), la construcción del propio edificio y las propiedades térmicas de los materiales empleados, la ocupación y su comportamiento, y subcomponentes como iluminación o sistemas de climatización, así como su funcionamiento y horarios.

\section{Fundamentos de la caracterización}

La base del modelo que se describe a continuación está precedida por la aplicación de las funciones de transferencia en el campo de la construcción (Stephenson \& Mitalas, 1971)(Ponsoda, Blanes, \& Bader, 2011). La principal ventaja de este modelo es su simpleza, pero siempre conservando el sentido físico. Existen diversos tipos de modelos de ingeniería: los muy elaborados y exhaustivos, o los que se pueden simplificar mediante el uso de diversas estrategias. El principal inconveniente es la necesidad de un gran número de datos de entrada, cuya precisión influye en gran medida en los resultados obtenidos. En cuanto a los modelos estadísticos (Coronado \& Álvarez, 2012; Pasut \& De Carli, 2012), son relativamente fáciles de desarrollar, pero exhiben una baja exactitud predictiva y son inflexibles.

La realidad es que los modelos de inteligencia artificial (Kreider \& Wang, 1991; Wang \& Kreider, 1992) son buenos para la resolución de problemas no lineales, haciéndolos muy aplicables para la predicción de energía en los edificios. El problema que se plantea es que son extremadamente complejos y requieren un gran número de datos históricos de funcionamiento.

Una de las preguntas que suelen plantearse es: ¿por qué utilizar el modelo de función de transferencia si existe un modelo general lineal que relaciona las diferentes variables? Hay varias razones detrás de la necesidad de los modelos de función de transferencia: La relación es casi instantánea y establecida a priori y la variable de entrada influye en la variable de salida, pero no viceversa. Además, no refleja una dependencia de la misma con respecto a sus valores pasados. La parte de la variable de salida, no detallada por la variable de entrada, se convierte en un término constante o ruido. Este término no puede verificar los datos dinámicos, por lo que no refleja la dependencia del sistema con lo que ha sucedido en los instantes anteriores.

Estos modelos se utilizan en todos los campos de la ciencia para evaluar las respuestas dinámicas. Si las entradas son variables controlables, estos modelos simulan y evalúan políticas alternativas. Si no es así, ofrecen la posibilidad de estudiar cómo determinados "escenarios", definidos por la posible evolución de la variable de excitación, afectan a la variable de respuesta.

Es importante que no se olvide el objetivo principal de este modelo, a través de la monitorización (en corto espacio de tiempo) de un edificio existente, se deben identificar los parámetros térmicos de un modelo para caracterizar el consumo de energía.

Asimilando las funciones de transferencia y la aplicación de su base, el modelo resultante para la caracterización térmica inversa es la ecuación (1). Donde: $f(t)$ es la variable objetivo. Y " $\mathrm{t}$ " es el tiempo. $Y_{i}$ son las variables independientes o excitaciones, con $\mathrm{i}=1, \ldots \mathrm{m} . a_{i j}$ son los coeficientes de ajuste de cada variable $Y_{i} . d_{k}$ son los denominadores o dependencias del variable objetivo con el pasado.

Las variables independientes deben ser elegidas de acuerdo con el sistema para caracterizar, y teniendo en cuenta su influencia sobre el mismo. Por ejemplo, las principales variables para la caracterización de los espacios son: Temperatura exterior y la radiación solar (que pueden aparecer juntas como sol-la temperatura del aire). Cargas internas: ocupación, iluminación, equipos.

Como se detalla más adelante, el procedimiento será el de establecer los valores de los denominadores, que se calculan a partir de las constantes de tiempo del sistema. Además, los numeradores se calculan para obtener el mejor ajuste con respecto a los datos medidos de la variable de destino, implementando un método de mínimos cuadrados.

Denominadores: Son los coeficientes que crean la dependencia de la variable correlacionada con la misma variable pero en momentos anteriores. Se calcula a partir de las constantes de tiempo (tau, $\tau_{i}$ ) del sistema. Estas constantes de tiempo se puede calcular, por ejemplo, con cualquier software de simulación, teniendo cuidado, para corregir su valor para el intervalo de tiempo elegido en la correlación. Teniendo en cuenta dos constantes de tiempo significativos (Fórmulas 2 y 3 )

$$
\begin{aligned}
& f(t)=\sum_{i=1}^{m}\left[\sum_{j=0}^{n} a_{i j} \cdot Y_{i}(t-j)\right]-\sum_{k=1}^{d} d_{k} \cdot f(t-k) \\
& d_{1}=-\left[\exp \left(-1 / \tau_{1}\right)+\exp \left(-1 / \tau_{2}\right)\right] \\
& d_{2}=\exp \left(-1 / \tau_{1}\right) * \exp \left(-1 / \tau_{2}\right)
\end{aligned}
$$

Numeradores: Son los coeficientes que multiplican las variables independientes, en este caso la excitación. Suponiendo que el criterio fija el flujo como negativo cuando es saliente, $a_{0}$ debe ser positivo. Los numeradores se calculan para obtener el mejor ajuste con respecto a los datos medidos de la variable de destino. Para ello, al menos se llevará a cabo una metodología de optimización de los cuadrados de los datos experimentales.

Las limitaciones que convierten esta identificación en una optimización son: Es un requisito de los signos de los coeficientes en función del criterio de signos para el flujo de calor seleccionado. Además, en algunos casos no se alternan los signos que pueden hacer negativo los numeradores segundo y cuarto. El ratio entre la suma de los numeradores y la suma de los denominadores está relacionado con la respuesta de estado estacionario del sistema de aumento de las temperaturas interior y exterior en un grado, y la dependiente de base estable en el tiempo elegido.

Por otra parte, los numeradores se calculan para obtener el mejor ajuste con respecto a los datos medidos de la variable de destino, implementando un método de mínimos cuadrados.

\section{Modelo integrado en base diaria}

Dadas las consideraciones anteriores, el producto final del modelo de caracterización en base diaria es una correlación que predice el consumo diario de energía térmica para el aire acondicionado y, por tanto, la temperatura dentro del edificio. 
Esto se hace mediante el uso de solo las variables climáticas (comprendidos en promedio diario sol-la temperatura del aire) y las horas de operación del sistema. En análisis climático se tienen algunas experiencias satisfactorias como por ejemplo (de la Flor, Francisco José Sánchez, Lissén, \& Domínguez, 2006; F. S. de la Flor \& Domínguez, 2004).

Existe una fuerte relación entre el uso de los equipos (demanda) y las condiciones internas (temperatura interior). Esto no se debe solo a las cargas internas de ocupación, iluminación y equipos (lleno de incertidumbre), sino a la operación HVAC: horarios, periodos de desconexión, intermitencia, fines de semana... Por lo tanto, es necesario modelar como una variable secundaria de la temperatura interior.

Así, el propio modelo se describe por dos correlaciones diferentes pero estrechamente relacionadas: uno para la demanda y otro para la temperatura interior. Cuando el sistema está apagado, la temperatura interior oscila libremente, basándose únicamente en las condiciones meteorológicas y las excitaciones del periodo anterior. Por lo tanto, el modelo para caracterizar la evolución de la temperatura interior, vinculado con el modelo principal para calcular el consumo diario de energía, requiere la partición en dos submodelos: Modelo para la temperatura interior en los días en los que el equipo funciona (por lo general de lunes a viernes).

Modelo para la temperatura interior en los días en los que el equipo no funciona (por lo general los fines de semana, vacaciones...). El modelo puede ser esquematizado, durante dos días genéricos, $t$ y $t+1$, del siguiente modo:

\section{Figura 2. Esquema general del modelo. Fuente: elaboración propia.}

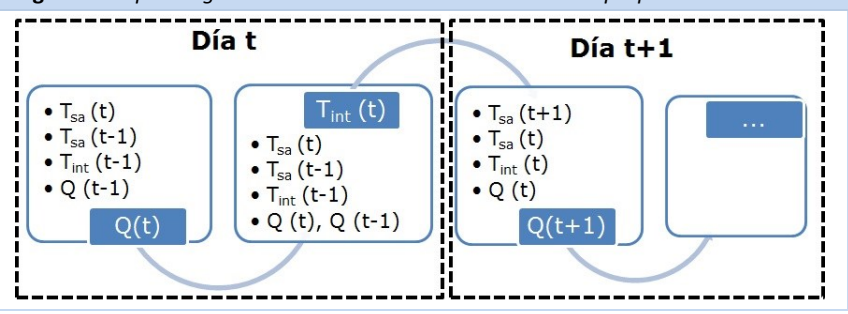

Con todo esto, el modelo genérico para predecir la demanda de un edificio se define con tres correlaciones como sigue:

$$
\mathrm{Q}(\mathrm{t})=\mathrm{a}_{0} \cdot \mathrm{T}_{\mathrm{sa}}(\mathrm{t})+\mathrm{a}_{1} \cdot \mathrm{T}_{\mathrm{sa}}(\mathrm{t}-1)+\mathrm{b}_{1} \cdot \mathrm{T}_{\text {int }}(\mathrm{t}-1)+\mathrm{d}_{1} \cdot \mathrm{Q}(\mathrm{t}-1)
$$

$\mathrm{T}_{\text {int ON }}(\mathrm{t})=\mathrm{a}_{0}{ }^{\prime} \cdot \mathrm{T}_{\mathrm{sa}}(\mathrm{t})+\mathrm{a}_{1}{ }^{\prime} \cdot \mathrm{T}_{\text {sa }}(\mathrm{t}-1)+\mathrm{d}_{1}{ }^{\prime} \cdot \mathrm{T}_{\text {int }}(\mathrm{t}-1)+\mathrm{b}_{0}{ }^{\prime} \cdot \mathrm{Q}(\mathrm{t})$

$+b_{1}{ }^{\prime} \cdot Q(t-1)$

$T_{\text {int OFF }}(t)=a_{0}{ }^{\prime \prime} \cdot T_{\text {sa }}(t)+a_{1}{ }^{\prime \prime} \cdot T_{\text {sa }}(t-1)+d_{1}{ }^{\prime \prime} \cdot T_{\text {int }}(t-1)+b_{1}{ }^{\prime \prime} \cdot Q(t-1)$

Donde: $Q(t)$ [kWh] es la demanda diaria de enfriamiento o calentamiento del sistema, calculada como la integral (suma) durante todo el período de operación. $T_{\text {int } o N}(t)\left[{ }^{\circ} \mathrm{C}\right]$ es la temperatura interior media diaria en el período de operación. $T_{\text {int } \text { OFF }}(t)\left[{ }^{\circ} \mathrm{C}\right.$ ] es la temperatura interior media diaria en el período de inactividad. $T_{s a}\left[{ }^{\circ} \mathrm{C}\right]$ es la temperatura sol-aire media diaria.
Los coeficientes $a$ y $b$ son los numeradores de cada correlación. Los coeficientes $d$ son los denominadores de cada correlación La variable $t$ es el tiempo en días. Los coeficientes se calculan a través de un proceso de identificación con restricciones. Tales restricciones establecen los signos de los numeradores y vienen definidos por la base de modelos de transferencia. En base diaria, son los siguientes: $a_{0}$ y $a_{0}{ }^{\prime}$ deben ser positivas, ya que representan la dependencia de las variables dependientes con la excitación climática.

Si $a_{1}$ y $a_{1}{ }^{\prime}$ son negativas, se revela la dependencia del modelo con el paso de tiempo anterior (debido al cambio de signo). Por el contrario, si son positivos podrían ser eliminadas del modelo. $b_{0}{ }^{\prime}$ será negativa: si la carga de refrigeración se considera positiva, ya que esto aumenta, la temperatura interior se reduce (manteniendo el resto de variables constante). Para la demanda de calefacción, con un signo negativo, si se aumenta, la temperatura interior aumenta también.

Por otra parte, $b_{1}{ }^{\prime}$ tiene un argumento idéntico al de $a_{1}$ y $a_{1}{ }^{\prime}$ : si es positiva, indica la dependencia del modelo con el paso de tiempo anterior. Denominadores $d_{1}$ y $d_{1}{ }^{\prime}$ serán siempre positivos. En el modelo de temperatura interior, la suma de los numeradores y denominadores debe ser uno. Esta restricción se refiere al rendimiento de estado estacionario del modelo físico.

\section{Validación mediante software de simulación energética de edificios DOE2-2}

Para demostrar la aplicación del procedimiento, se aplica a una oficina de un gran edificio. Pertenece a la base de datos de grupo de Termotecnia de la Universidad de Sevilla, y está implementado en la herramienta CALENER GT (herramienta oficial de calificación energética de edificios del gobierno de España), y es simulado por el motor de cálculo DOE2-2, lo que garantiza la calidad de las mediciones. Se decide utilizar DOE2-2 (James J. Hirsch \& Associates \& Lawrence Berkeley National Laboratory, 2001) por ser una herramienta consolidada que implementa modelos reales de los equipos.

Se elige un edificio aislado de oficinas por las siguientes razones: Su climatización es independiente del resto del edificio, tiene un sistema autónomo que controla la temperatura y satisface su demanda, permitiendo de este modo la inversión térmica sucesiva. Ser un espacio único que sirve como un "laboratorio" para probar los posibles cambios en el horario, ajuste de temperatura de consigna, las características de la epidermis y para verificar que el procedimiento es válido.

Las siguientes imágenes muestran tanto el edificio como el área bajo estudio. A lo largo de la experimentación llevada a cabo, las características de la epidermis de la zona de estudio modelo ha cambiado, evidenciando una situación sin lagunas en el caso que se presenta, con su recinto altamente esmaltado (recinto de vidrio tiene una superficie mayor que $80 \%$.), orientado al sur.

La oficina en estudio tiene una superficie total de $50 \mathrm{~m}^{2}$. Su sistema de HVAC está dimensionado para cubrir la punta de carga de calefacción y refrigeración para mantener una temperatura interior de 20 y $25^{\circ} \mathrm{C}$ respectivamente. Durante la simulación, algunas variables sobre una base horaria referidas al sistema, la zona y la climatología, que son necesarias para el 
desarrollo del modelo, se extraen de DOE2-2. Estas variables son: refrigeración / calefacción de carga suministrada por la batería (total y latente), tanto en modo refrigeración y calefacción, la temperatura interior, la temperatura exterior y la humedad, radiación solar y velocidad del viento. Con estos datos, el procedimiento se aplica a un periodo representativo de la estación de refrigeración (2-22 de julio). En ese caso, la correlación se prueba con un periodo de validación (de 0723 hasta 08 31).

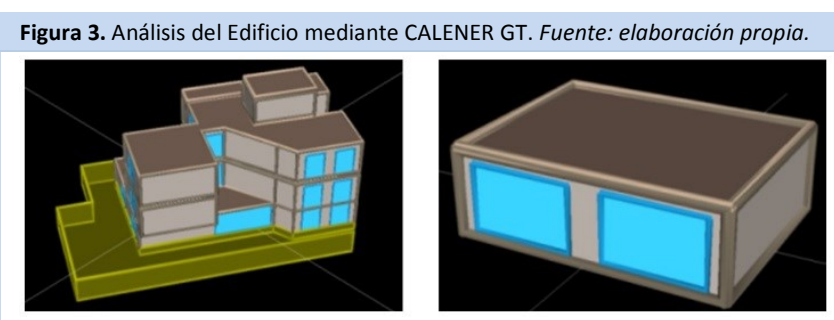

Existen muchos autores con publicaciones sobre el diagnóstico térmico, especialmente en los años 90 y posterior, pero destaca el trabajo de A. Rabl (Rabl, 1988; Rabl \& Norford, 1991; Rabl \& Rialhe, 1992) por su visión del paisaje como real. Según A. Rabl, atendiendo a edificios ocupados, pueden ser aceptados errores en la identificación de $20 \%$ en el coeficiente global de transmisión de la epidermis, $50 \%$ en solar y $50 \%$ en la constante de tiempo dominante del sistema. Por lo tanto, es posible aceptar una incertidumbre $20 \%$ en el consumo de energía.

Por último, el cálculo de la demanda mensual estimada como la suma de las exigencias diarias, y comparándola con la demanda simulada conocida, los errores relativos cometidos resultan ser más que aceptable tanto para el período de base y el período de validación: $1,6 \%$ de julio, $8,4 \%$ para el mes de agosto.

Figura 4. Simulado (azul - DOE2.2) y demanda correlada (rojo) en el periodo de refrigeración (día-mes). Error promedio diario cometido $5.1 \%$, con un valor máximo de error relativo de $18.1 \%$. Fuente: elaboración propia.

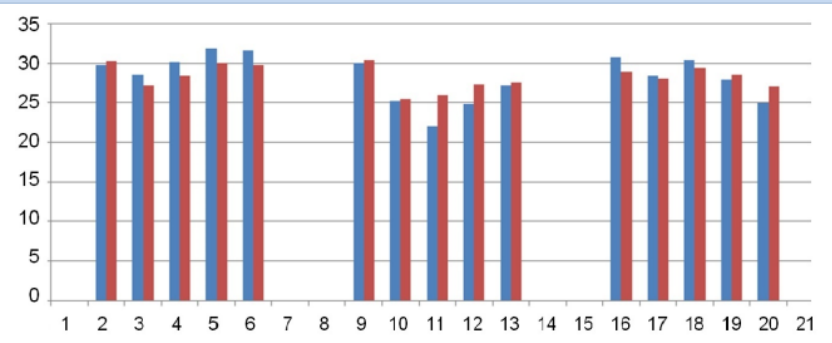

Figura 5. Evolución de temperaturas tipo en la impulsión y retorno de las unidades de climatización del espacio a caracterizar. Fuente: elaboración propia.

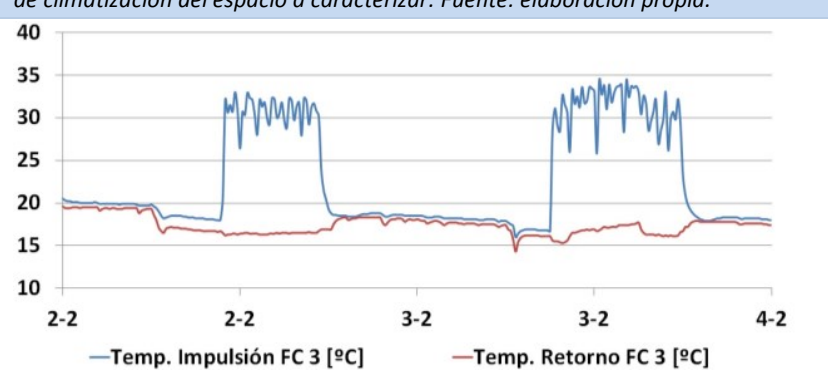

-Temp. Impulsión FC 3 [ำ] -Temp. Retorno FC 3 [으

\section{Resultados del edificio a estudio: Palacio de La Madraza}

\section{Monitorización}

El análisis se centra en la Sala de Administración del edificio Palacio de La Madraza por su carácter intensivo, periodicidad en los horarios y factor de gasto energético considerable en relación a las demás salas del edificio, destinadas a un uso puntual.

El Sistema de Gestión Integrada de la Universidad de Granada, implementada en el edificio, permite recoger valores tales como el estado del sistema de climatización además de temperaturas de referencia. Cumplimentando estas variables, se decide colocar una batería de data loggers para recoger las temperaturas de impulsión y retorno en cada uno de los fancoils y enriquecer, de este modo, las variables objeto de estudio.

De modo paralelo se establece un control por parte de los usuarios del recinto, en el que colaboran con las pautas de uso, constituyéndose así una comunicación directa mediante cuestionarios y control de movimientos que permite ajustar las condiciones de uso. Se disponen data loggers recogiendo temperaturas de impulsión y retorno de los fancoils en la Sala de Administración en un intervalo de recogida de datos de diez minutos, cuyo objetivo es obtener de forma diferida la energía suministrada al recinto debido al régimen de caudal constante en el que funcionan los elementos de climatización. Del mismo modo, se colocan tres sensores que recogen la temperatura ambiente de la sala, correspondiendo cada una de las medidas aportadas a un tercio del recinto y dispuestos a distintas alturas, dando la información de un modo zonificado y estratificado. Los modelos utilizados fueron mini data logger Testo T174T y data logger Testo 175T1, así como un data logger Testo $\mathrm{T} 175 \mathrm{H} 1$ para recoger la humedad de la sala.

\section{Discusión}

\section{Datos medidos}

A partir de la medición de las temperaturas de aire de retorno e impulsión de los fancoils instalados en la sala, y registrando su periodo de operación mediante el sistema de gestión instalado, se puede calcular la energía suministrada en cada intervalo de medida con la siguiente fórmula:

$E_{i j}=C p \cdot M \cdot\left(T_{I M P i j}-T_{R E T i j}\right) \cdot D j$

Dónde Eij es la energía en Julios suministrada por el fancoil $i$ en el intervalo j. Cp $\left[\mathrm{J} / \mathrm{kg}^{\circ} \mathrm{C}\right]$ es el calor específico del aire, calculado para la temperatura media interior del periodo de operación y una humedad relativa del $50 \%$. M [kg/s] es el caudal de aire impulsado por el fancoil que, puesto que son a caudal constante, es el mismo para todos los sistemas. Dj [s] es el intervalo de medida en segundos. En este caso se ha elegido 10 minutos. A la par, la energía diaria suministrada por el fancoil i se obtiene sumando durante el periodo de operación la energía suministrada en cada paso de cálculo. A modo de ejemplo se presenta la evolución de temperatura para dos días de operación del fancoil tres. A la par la energía entregada en estos dos días de operación. 
Figura 6. Temperaturas promedio en el espacio a caracterizar: azul temperatura promedio interior $\left[{ }^{\circ} \mathrm{C}\right]$, rojo temperatura del aire exterior $\left[{ }^{\circ} \mathrm{C}\right]$. Fuente: elaboración propia.

30

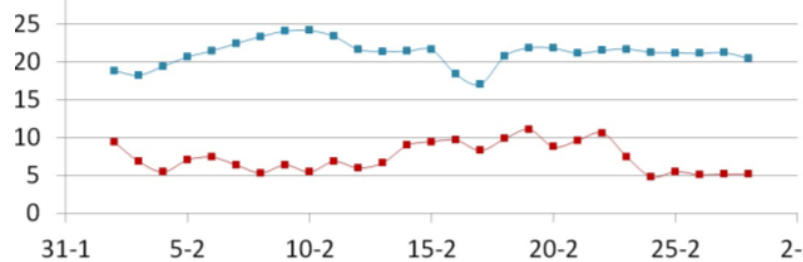

Identificación inversa del modelo de caracterización

Al trabajar con una base diaria, las variables de trabajo son temperaturas promediadas o energía integrada en la base de tiempo de trabajo. Las dos variables objetivo son: la temperatura interior promedio, como representación de la inercia, comportamiento de la sala frente a las excitaciones y periodos de operación; y la energía suministrada como el producto requerido para la evaluación de ahorros y la verificación posterior.

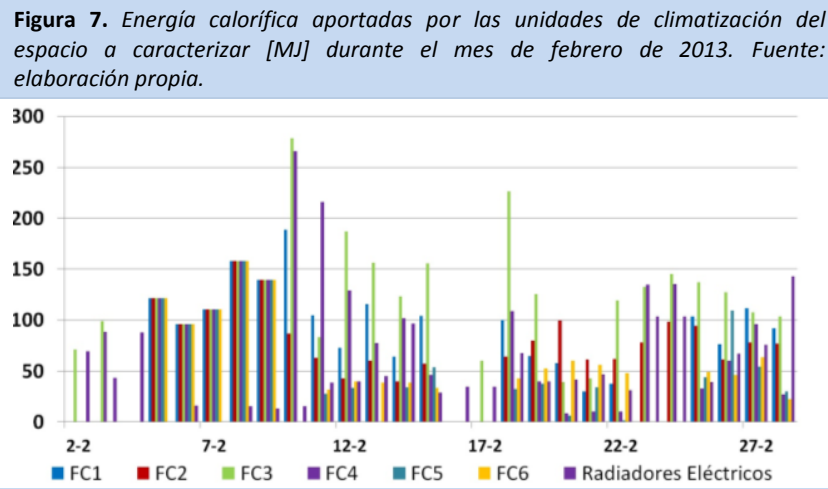

Sorprende el hecho de que no aparecen unos periodos de no consumo, tal y como se esperaba, correspondientes a los periodos de fin de semana en los que la sala debería operar bajo los mínimos de seguridad requeridos.

Es por este motivo que se anidan dos modelos para caracterizar la evolución de la temperatura y de la carga: un modelo correspondiente a los periodos de funcionamiento normales con demandas diarias superiores a 300 MJ (modelo básico); y otro con el que se coge el funcionamiento a carga parcial (modelo parcialidad). Esta decisión se fundamenta en el hecho de que los radiadores eléctricos usados de apoyo en la sala operan sin controlar la temperatura y bajo demanda de usuario, sin guardar relación con la evolución de la temperatura exterior (véase gráficas anteriores).

Por consiguiente, al modelo dúplex y anidado que se ha presentado se le identifican los coeficientes a partir de los datos experimentales que se tienen, de tal forma que se consiga el mejor ajuste del modelo no lineal presentado. De esta forma quedaría caracterizada la demanda energética de la sala de administración, pudiéndose usar el modelo como función predictiva.

La energía es la verdadera variable objetivo, por lo que se debe cuidar el control de la incertidumbre, es decir, es admisible un $25 \%$ de error incluso un $30 \%$ mientras sea un error controlado.
Es por esto que entre las líneas rojas se dibuja el $25 \%$ de error, y que la mayor parte de los puntos se sitúan contenidos en su interior.

Además, hay que añadir que existen una serie de puntos, correspondientes a los días de febrero en los que se alcanza una demanda pico 1.5 veces superior a la demanda típica del espacio. Estos picos quedan explicados por la conexión de los radiadores eléctricos por parte de los usuarios cuando los fancoils trabajan a plena carga, es decir, exceso de demanda por inexistencia de control térmico.

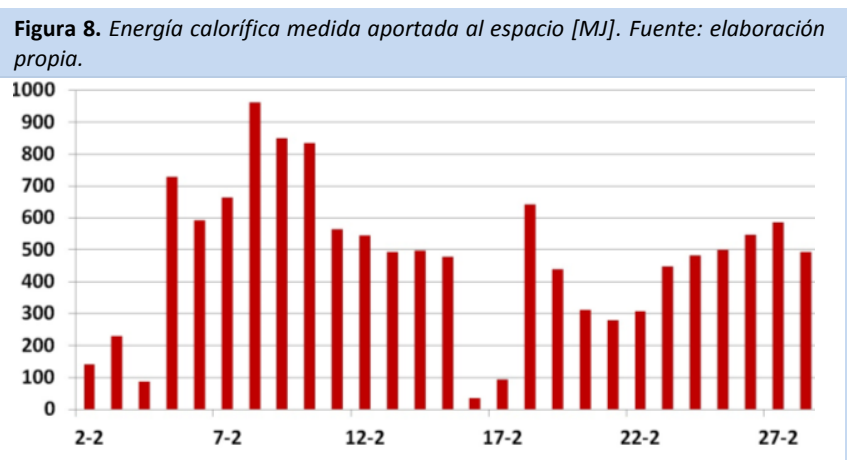

Figura 9. Energía calorífica medida [MJ] frente a modelada [MJ]. Fuente: elaboración propia.

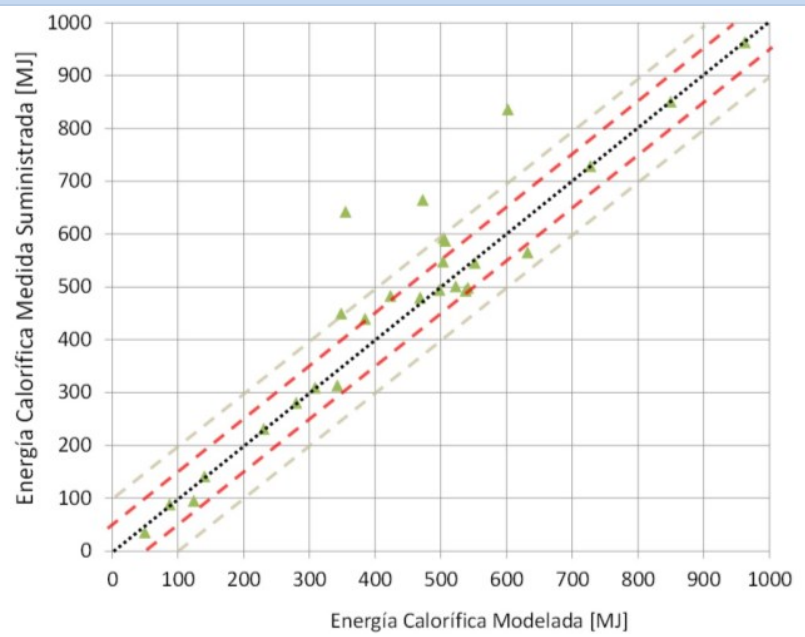

Figura 10. Temperatura del aire interior promedio medida $\left[{ }^{\circ} \mathrm{C}\right]$ frente a modelada [ํㅡ. Fuente: elaboración propia.

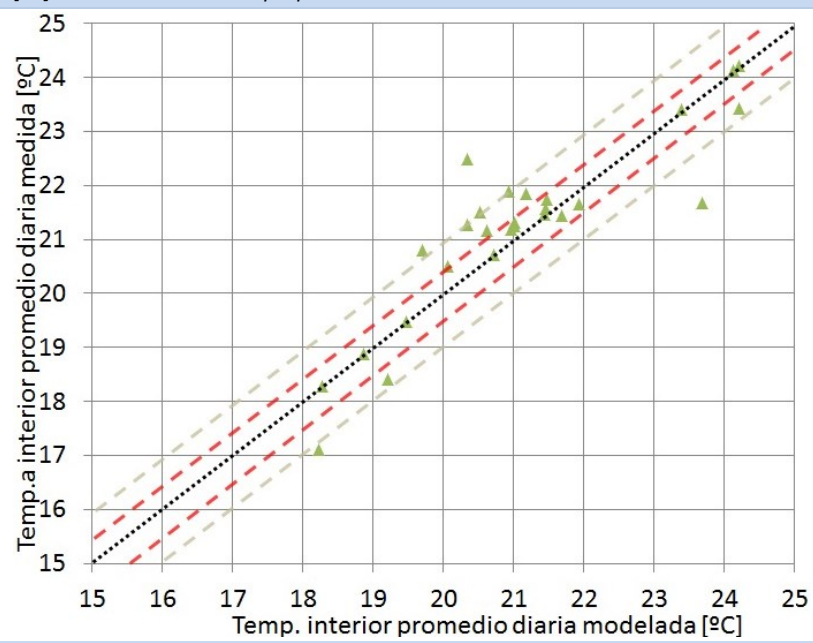


La temperatura, aunque es una variable auxiliar, es clave en la calidad del modelo, ya que encierra la esencia física de la respuesta de la sala frente a las excitaciones climáticas y del equipo de acondicionamiento. Es por esto que se puede ver como los errores que aparecen son en valor promedio cercanos a 0.5 ㅇ C (línea roja), aunque aparecen controlados y como máximo en los puntos representativos se alcanza $1^{\circ} \mathrm{C} C$ (línea gris).

\section{Conclusiones}

Además de para la inspección/diagnosis del edificio y sus sistemas, se requiere una monitorización energética en una primera instancia de duración corta, y probablemente, tras la implantación del proyecto de rehabilitación, de carácter fijo. Como ya se ha comentado, el consumo procurará ser eficiente a partir de las medidas implementadas, pero también inteligente, racionalizando con control y gestión la energía consumida.

Es importante destacar la diferencia que aparece en el concepto de servicios con medición "Smart" e "Intelligent", ya que a priori ambos destacan la inteligencia del control realizado, pero existen diferencias: "Smart" ha sido usado en servicios eléctricos, de agua y gas, considerando una lectura regular y detallada, que crea una comunicación del gestor y de la utilidad (uso final), con una monitorización y lectura automatizada. "Intelligent" en adicción a lo anterior, analiza estas observaciones e identifica decisiones o sucesos que permiten la optimización del servicio. A su par, existe una comunicación entre el gestor y el usuario final en las dos direcciones.

Una vez realizado el inventario energético y establecido el proyecto de rehabilitación energética, es imprescindible estimar e informar de los ahorros. Al implantarse una medida de mejora en un edificio, cambia su comportamiento y se pierde la situación de referencia (línea base). Para poder calcular el ahorro conseguido con dicha mejora, debe compararse el consumo de energía actual y el que se habría tenido si la medida no se hubiera llevado a cabo. Así, un modelo predictivo permite proyectar el uso de energía en el edificio desde un periodo de referencia, que puede ser medido, a un periodo posterior. Esta proyección requiere el ajuste de la línea base a diferentes condiciones de clima, ocupación u otras variables que puedan afectar al consumo de energía.

Dado que los modelos actuales en general son complejos y de difícil aplicación, en el presente documento se ha propuesto un nuevo modelo. La predicción se realiza en dos niveles: horario, con un modelo derivado de las funciones de transferencia aplicadas a la edificación, y diario, con un modelo simplificado a partir del anterior.

A modo de ejemplo, se realiza un estudio de aplicación real para la estación de calefacción, extrayendo las siguientes conclusiones: En cuanto a errores diarios, éstos se mantienen en una franja alrededor del $25 \%$, tanto en el periodo base como en el periodo de validación. Si se calcula el error promedio diario, se tiene que para el periodo base es cercano al $5 \%$, mientras que para el periodo de validación es aproximadamente un $10 \%$. Si se calcula el error en base mensual, éste es siempre menor al $10 \%$.
Es decir, con el modelo diario propuesto, mediante un procedimiento sencillo, semiautomático gracias a las herramientas proporcionadas, basado en mediciones tan solo de consumo energético, temperatura interior $y$ datos climáticos, se consigue una predicción suficientemente precisa y se demuestra su aplicabilidad y amplio rango de validez.

Esta tecnología supone un salto de alto nivel innovador, ya que a partir de un modelo sencillo de caracterización, identificado con datos reales, se va a tener la esencia física del sistema monitorizado. Por consiguiente, se tiene el comportamiento real, y más aún, se tiene una herramienta sencilla, que excitada con las entradas que se quieran, devuelve la respuesta supuesta real del sistema. Es por esto, que podríamos estimar el consumo que tendría el edificio en 2020 si se tiene el clima. Esta herramienta permite gestión energética, control, y sobre todo, verificación de ahorros.

\section{Agradecimientos}

Los autores de este artículo agradecen de manera especial a Dña. Begoña Escobar, vicerrectora de Infraestructuras y Campus de la Universidad de Granada, y al director de la Oficina de Energía e Instalaciones, don Antonio Espín Estrella, por hacer factible el desarrollo de esta investigación. Al equipo de investigación de la Cátedra de Termotecnia de la Universidad de Sevilla (España), en especial a los catedráticos D. Servando Álvarez Domínguez y D. José Luis Molina Félix, por su implicación y dedicación al trabajo. Al departamento de Motores Térmicos de la Universidad de Cádiz (España), por colaborar cediendo el equipo de monitorización portátil, y su siempre disponibilidad. Este trabajo ha sido realizado y financiado en el marco del Plan de Control y Monitorización de Edificios del Vicerrectorado de Infraestructuras y Campus de la Universidad de Granada. 


\section{Referencias}

Coronado, M. T., \& Álvarez, S. (2012). Rehabilitación energética de edificios existentes: Diagnóstico y verificación de ahorros. Modelo simplificado de caracterización inversa integrado. PFC. Sevilla: Publicaciones de la Universidad de Sevilla.

De la Flor, Francisco José Sánchez, Lissén, J. M. S., \& Domínguez, S. Á (2006). A new methodology towards determining building performance under modified outdoor conditions. Building and Environment, 41(9), 1231-1238. doi: 035.

De la Flor, F. S., \& Domínguez, S. A. (2004). Modelling microclimate in urban environments and assessing its influence on the performance of surrounding buildings. Energy and Buildings, 36(5), 403-413.

Hai-xiang, Z., \& Magoulès, F. (2012). A review on the prediction of building energy consumption. Renewable and Sustainable Energy Reviews, 16 (6), 3586-3592.

Kreider, F., \& Wang, X. (1991). Artificial neural networks demonstration for automated generation of energy use predictors for commercial buildings. ASHRAE Transactions, 97.

Pasut, W., \& De Carli, M. (2012). Evaluation of various CFD modelling strategies in predicting airflow and temperature in a naturally ventilated double skin façade. Applied Thermal Engineering, 37(0), 267274. doi:10.1016/j.applthermaleng.2011.11.028.

Ponsoda, E., Blanes, S., \& Bader, P. (2011). New efficient numerica methods to describe the heat transfer in a solid medium. Mathematical and Computer Modelling, 54(7-8), 1858-1862. doi:10.1016/j.mcm.2010.11.067.

Rabl, A. (1988). Parameter estimation in buildings: Methods for dynamic analysis of measured energy use. Journal of Solar Energy Engineering, Transactions of the ASME, 110(1), 52-66.

Rabl, A., \& Norford, L. K. (1991). Peak load reduction by preconditioning buildings at night. International Journal of Energy Research, 15(9), 781798.

Rabl, A., \& Rialhe, A. (1992). Energy signature models for commercial buildings: Test with measured data and interpretation. Energy and Buildings, 19(2), 143-154.

Stephenson, D. G., \& Mitalas, G. P. (1971). Calculation of heat conduction transfer functions for multi-layer slabs. ASHRAE transactions, 77 (2), 117-126.

Wang, X.A. \& Kreider, J.F., Improved artificial neural networks for commercial building energy use prediction, Solar Engineering ASM 1, vol. 1, 1992, p.361-366.

Wouters, L., \& Vandaele, L. (1995). PASLINK final activity report. Bruselas: Publications of European Comission. 